\title{
Translation and linguistic validation of the EORTC QLQ-PAN26 questionnaire for assessment of health-related quality of life in patients with pancreatic cancer and chronic pancreatitis into isiXhosa and Afrikaans
}

\author{
KJ Gqada, ${ }^{1,2}$ (D) U Kotze, ${ }^{(1 D}$ V Soldati, ${ }^{1,2}$ (D) C Kloppers, ${ }^{1,2}$ (D) J Krige, ${ }^{1,2}$ (D) E Jonas ${ }^{1,2}$ (iD) \\ ${ }^{1}$ Department of Surgery, Faculty of Health Sciences, University of Cape Town, South Africa \\ ${ }^{2}$ Surgical Gastroenterology Unit, Groote Schuur Hospital, South Africa
}

Corresponding author,email: eduard.jonas@uct.ac.za

\begin{abstract}
Background: Health-related quality of life (HRQOL) parameters have become important components in the holistic management of pancreatic ductal adenocarcinoma (PDAC) and are now increasingly incorporated in treatment protocols. The European Organisation for Research and Treatment of Cancer (EORTC) pancreatic cancer specific questionnaire (QLQ-PAN26) has also been validated for chronic pancreatitis (CP). The objective was to translate the EORTC QLQPAN26 questionnaire into and validate it for isiXhosa and Afrikaans.

Methods: Following the EORTC translation procedure, two forward translations of the English version into isiXhosa and Afrikaans were performed independently by two language practitioners for each language, followed by reconciliation of disagreements. A back translation of the reconciled version into English by a second pair of language practitioners was done. The results of all the steps were summarised with comments in a report for review by the EORTC translation unit. After proofreading by an external proof-reader chosen by the translation unit, pilot testing was performed on a cohort of ten isiXhosa patients and ten Afrikaans patients with PDAC or chronic pancreatitis. Results were summarised in a pilottesting report, and the final version approved by the translation unit.

Results: Thirteen patients diagnosed with PDAC and seven with CP were included in the study. The questionnaire was completed electronically $(n=12)$ or on paper $(n=8)$. Median age in the isiXhosa group was 53.7 (range $41-63)$ and in the Afrikaans group 60.9 (range 35-79). Questions 31-54 had a 100\% completion rate, while 35\% of respondents did not complete Q55 and Q56. Internal consistency was satisfactory in isiXhosa (alpha $=0.88)$ and Afrikaans $($ alpha $=0.89)$.

Conclusion: The EORTC QLQ-PAN26 used in patients with PDAC and CP has been translated and linguistic validation performed in isiXhosa and Afrikaans. Availability of a questionnaire in patients' mother tongue should increase the validity of results.

Keywords: health-related quality of life, pancreatic ductal adenocarcinoma, questionnaire translation
\end{abstract}

\section{Introduction}

Pancreatic ductal adenocarcinoma (PDAC) is one of the most lethal malignant neoplasms worldwide, ranking seventh as a cause of cancer mortality. ${ }^{1}$ Although surgical resection offers potential cure, most patients have advanced disease at presentation. Indeed, most PDACs have a poor prognosis, with an overall five-year survival of less than $10 \%{ }^{2}$ Both the disease and the treatment may also have significant morbidity. ${ }^{3}$ Morbidity, mortality and survival rates are frequently used as outcome measures in PDAC. However, there are other important subjective elements of patients' quality of life (QOL) that impact on wellbeing. These less frequently reported elements include aspects of employment, social activities, emotional functioning, finances, family and sexual life. ${ }^{4}$

Health-related (HR) QOL are now regarded as an important component in the holistic management of PDAC and are now increasingly incorporated in treatment protocols and reported in clinical research trials. ${ }^{5}$ The European Organisation for Research and Treatment of Cancer (EORTC) has developed a PDAC-specific questionnaire (QLQ-PAN26) (Table I) which is used in conjunction with the generic core QLQ-C30 cancer questionnaire (Q 1-30), which already has isiXhosa and Afrikaans translations. ${ }^{6,7}$ The questionnaire incorporates a number of symptom scales relevant to PDAC and comprises 26 questions (Q 31-56), each with four response options which facilitate structured and quantifiable HRQOL measurements. In 2005, the questionnaire was also validated for chronic pancreatitis (CP). ${ }^{8}$

The worldwide use of the standardised questionnaire allows comparison of results from different HRQOL studies. Translation and validation of questionnaires into indigenous and local languages have enabled their wider applicability, allowing inclusion of populations that traditionally have 


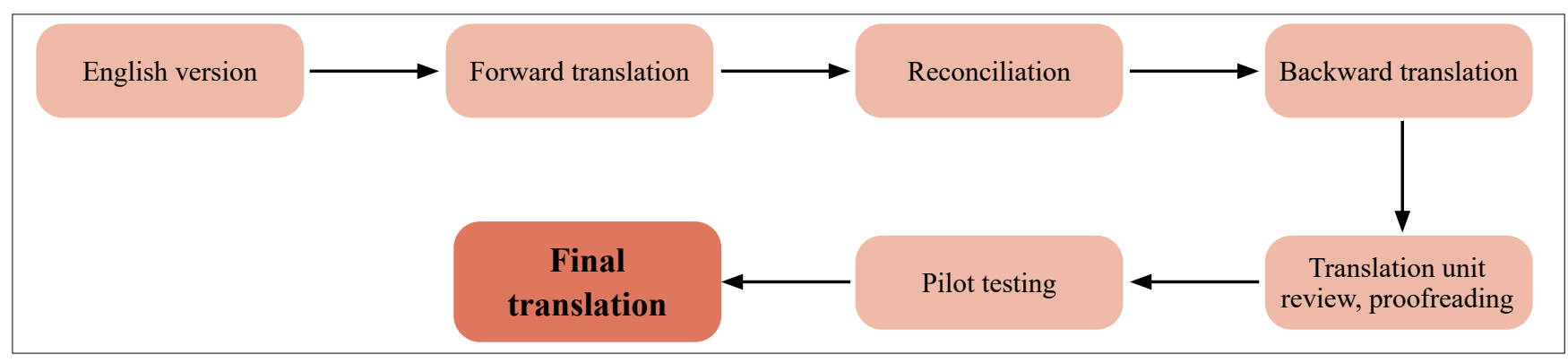

Figure 1: The EORTC translation procedure

been under-represented in research studies. The translation and validation process can be complex, as there are significant conceptual, semantic and linguistic differences which may complicate language translations. ${ }^{9}$ To date the QLQ-PAN26 has not been translated into any indigenous African languages. We report the translation and validation of the QLQ-PAN26 into the indigenous South African languages isiXhosa and Afrikaans using the prescribed EORTC translation procedure. ${ }^{10}$

\section{Methods}

Collection, analysis, and translation of QOL data were performed. Study data were collected prospectively and managed using REDCap electronic data capture tools hosted at the University of Cape Town. ${ }^{11,12}$ The EORTC translation procedure manual is available to researchers and the protocol is briefly outlined in Figure $1 .{ }^{10}$ The translation unit confirmed that isiXhosa and Afrikaans translations did not exist.

Two forward translations of the English version into isiXhosa and Afrikaans were performed independently by two language practitioners who were native speakers of the indigenous languages and also proficient in English. The two language practitioners compared the translations and merged them into a reconciled version. Identical translations were accepted. Where the translations differed by single words with similar meanings that were interchangeable without changing sentence structure, the translators agreed on the word they concluded would be the most widely understood based on anecdotal local engagement. Where translations had different sentence structure and reviewers agreed that the versions had the same meaning, the simplest version was used. The formulations of the four-level Likert scale for the languages were from previous translations, which are universally used throughout EORTC questionnaires. This was followed by independent back translation of the reconciled version into English by a second pair of language practitioners. During these steps, comments were made by the reviewers on semantic differences in the vocabularies of the respective languages, cultural meanings, appropriateness and colloquialisms. This revolved predominantly around English words which have no direct surrogates in the target indigenous languages. As a result, the back translations, when taken verbatim, were often not identical to the original English versions. For example, when referring to flatulence, the isiXhosa version would read, "umoya" and Afrikaans, "winderigheid". Directly back translated, this could be read, "wind" or "spirit". However, within the clinical context the meaning is clear. Another anecdote would be around the term "sex". We agreed to the term "kwezokwabelana ngesondo" in isiXhosa, and "seks" in Afrikaans. These terms were considered to be formal and, especially in the case of the isiXhosa version, somewhat archaic. However, they were selected in favour of other colloquial terms, which may be in more common use but may be less acceptable. The results of all the steps (forward translation, reconciliation and backward translation with comments) were summarised in a report for review by the EORTC translation unit.

After resolving issues raised by the translation unit, the preliminary translation was proofread by an external proofreader appointed by the translation unit. All suggestions were analysed and discussed until consensus was reached. After completion, the questionnaire was pilot tested for linguistic validation in ten isiXhosa patients and ten Afrikaans patients with PDAC or CP. Consecutive patients who identified their language of preference as either Afrikaans or isiXhosa were recruited to have the assessment performed using the translated questionnaire. Patient comments on any difficulties they experienced with the questionnaire and suggested improvements were summarised in a pilot-testing report which was assessed by the EORTC translation unit before final approval of the translated questionnaire.

There are a total of seven multi-item symptom scales in the QLQ-PAN26, namely pancreatic pain (questions 31, 33, 34, 35 ), gastrointestinal symptoms (questions 36, 37), altered bowel habits (questions 46, 47), hepatic (questions 44, 45 ), body image (questions 48, 49), healthcare satisfaction (questions 53, 54), and sexuality (questions 55, 56) (Table I). To further validate, the internal consistency of each of these scales and of the entire questionnaire was calculated using the Cronbach's alpha co-efficient. A co-efficient of $>0.7$ was considered acceptable.

\section{Results}

The pilot groups consisted of two females and eight males in the isiXhosa-speaking group and five females and five males in the Afrikaans-speaking group. Median age was 53.7 (range 41-63) in the isiXhosa group and 60.9 (range $35-79)$ in the Afrikaans group. Six patients $(60 \%)$ in the isiXhosa group and eight patients $(80 \%)$ in the Afrikaans group had completed secondary school qualification. Regarding socioeconomic status, all patients in the cohort were classified at hospital registration as being either not formally employed, employed but having an annual income of less than 70000 ZAR per annum, or receiving government welfare in the form of grants. Thirteen patients had PDAC and seven had CP. Patients completed the questionnaire either electronically $(n=12)$ or on paper $(n=8)$ and was either self-administered $(n=16)$ or by interview $(n=4)$. Overall, the questionnaire completion rate was excellent. Questions 31-54 had a 100\% completion rate. Questions 55 and 56, which address sexuality, were exceptions, with $35 \%$ 
Patients sometimes report that they have the following symptoms or problems. Please indicate the extent to which you have experienced these symptoms or problems during the past week. Please circle the number that best applies to you.

31. Have you had abdominal discomfort?

32. Did you have a bloated feeling in your abdomen?

33. Have you had back pain?

34. Did you have pain during the night?

35. Did you find it uncomfortable in certain positions (e.g. lying down)?

36. Were you restricted in the types of food you can eat as a result of your disease or treatment?

37. Were you restricted in the amounts of food you could eat as a result of your disease or treatment?

38. Did food and drink taste different from usual?

39. Have you had indigestion?

40. Were you bothered by gas (flatulence)?

41. Have you worried about your weight being too low?

42. Did you feel weak in your arms and legs?

43. Did you have a dry mouth?

44. Have you had itching?

45. To what extent was your skin yellow?

46. Did you have frequent bowel movements?

47. Did you feel the urge to move your bowels quickly?

48. Have you felt physically less attractive as a result of your disease and treatment?

During the past week:

49. Have you been dissatisfied with your body?

50. To what extent have you been troubled with side-effects from your treatment?

51 . Were you worried about your health in the future?

52. Were you limited in planning activities in advance (e.g. meeting friends)?

53. Have you received adequate support from your health care professionals?

54. Has the information given about your physical condition and treatment been adequate?

55. Have you felt less interest in sex?

56. Have you felt less sexual enjoyment?

\begin{tabular}{|c|c|c|c|}
\hline Not at all & A little & Quite a bit & Very much \\
\hline 1 & 2 & 3 & 4 \\
\hline 1 & 2 & 3 & 4 \\
\hline 1 & 2 & 3 & 4 \\
\hline 1 & 2 & 3 & 4 \\
\hline 1 & 2 & 3 & 4 \\
\hline 1 & 2 & 3 & 4 \\
\hline 1 & 2 & 3 & 4 \\
\hline 1 & 2 & 3 & 4 \\
\hline 1 & 2 & 3 & 4 \\
\hline 1 & 2 & 3 & 4 \\
\hline 1 & 2 & 3 & 4 \\
\hline 1 & 2 & 3 & 4 \\
\hline 1 & 2 & 3 & 4 \\
\hline 1 & 2 & 3 & 4 \\
\hline 1 & 2 & 3 & 4 \\
\hline 1 & 2 & 3 & 4 \\
\hline 1 & 2 & 3 & 4 \\
\hline 1 & 2 & 3 & 4 \\
\hline Not at all & A little & Quite a bit & Very much \\
\hline 1 & 2 & 3 & 4 \\
\hline 1 & 2 & 3 & 4 \\
\hline 1 & 2 & 3 & 4 \\
\hline 1 & 2 & 3 & 4 \\
\hline 1 & 2 & 3 & 4 \\
\hline 1 & 2 & 3 & 4 \\
\hline 1 & 2 & 3 & 4 \\
\hline 1 & 2 & 3 & 4 \\
\hline
\end{tabular}

Table II: Internal consistency for the overall questionnaire and for the individual multi-item symptom scales

\begin{tabular}{lcc} 
Table II: Internal consistency for the overall questionnaire and for the individual multi-item symptom scales \\
\hline Symptom scale & Cronbach's alpha isiXhosa & Cronbach's alpha Afrikaans \\
\hline Overall & 0.88 & 0.89 \\
Pancreatic pain & 0.76 & 0.85 \\
Gastrointestinal & 0.89 & 0.595 \\
Altered bowel habits & 0.24 & 0.41 \\
Jaundice & 0.40 & 0.58 \\
Body image & 0.98 & 0.77 \\
Healthcare satisfaction & 0.73 & 0.765 \\
Sexuality & 0.93 & 0.94
\end{tabular}

of respondents not providing an answer. These included two patients from the isiXhosa group (20\%) and five patients (50\%) from the Afrikaans group. Some patients said that they were not sexually active therefore the questions did not apply to them. Others indicated that they were uncomfortable commenting on what they referred to as private matters. The non-responders to these two questions were all female.
Internal consistency was satisfactory for the questionnaire as a whole in both isiXhosa (alpha $=0.88)$ and Afrikaans (alpha $=0.89$ ). Cronbach's alpha for each multi-item symptom scale is recorded in Table II.

\section{Discussion}

HRQOL assessments have evolved from instruments for assessing patient-wellbeing to useful clinical tools estimating 
surgical risks and predicting outcome. ${ }^{13}$ To optimise their use, HRQOL surveys must be clearly understandable to broad categories of users and their patients, which requires reliable translations into indigenous languages with deference to unique cultural and socioeconomic aspects. ${ }^{9}$ South Africa has eleven official languages, of which three Afrikaans, isiXhosa and English - are the predominant languages spoken in the Western Cape Province.

Most published HRQOL studies originate from first-world institutions and assess patient cohorts from well-resourced healthcare systems, where mechanisms are in place for comprehensive multi-level care of patients, including palliative services. In these settings, HRQOL is used as a tool to optimise individual patient's management. In developing countries, the majority of patients do not have access to this level of care. Furthermore, a significant proportion of patients are indigent and socio-economically vulnerable. This also manifests in the two-tiered South African healthcare system where patients that are not able to afford private health insurance who are predominantly treated in the public healthcare service. This may influence responses to selected questions ( $Q$ 51-53) and reveal deficiencies such as lack of food or absence of a primary carer, factors infrequently encountered in well-resourced countries.

Another aspect that needs consideration is cultural factors that may influence the answers to certain questions, for example, the lower response rate in sexuality-related questions ( $\mathrm{Q} 55,56)$ observed in this study. The reasons are likely related to religion and cultural propriety. The dominant religions are Christian and Muslim faiths where, especially with conservative denominations, there is reluctance to openly discuss sexuality. From the fact that all non-responders were female, we infer a possible cultural influence which preserves sexual conservatism especially in women.

Our results show excellent internal consistency in both translated versions of the questionnaire. We found lower Cronbach's alpha scores in the gastrointestinal, altered bowel habits and hepatic scales deviations attributed to small patient numbers and only two questions per scale. This is consistent with pilot studies of other translations. ${ }^{14}$

\section{Conclusion}

The EORTC QLQ-PAN26 used in patients with PDAC and $\mathrm{CP}$ has been translated into isiXhosa and Afrikaans and linguistic validation achieved through pilot testing. Translation into patients' mother tongue should increase validity of results during quantitative assessment of HRQOL. Results have to be interpreted in the context of unique cultural and religious aspects. In addition, the influence of socio-economic status on general wellbeing may impact on results, particularly in developing countries.

\section{Conflict of interest}

The authors declare no conflict of interest.

\section{Funding source}

KJ Gqada received a research grant via the DST-NRF Innovation Masters Scholarship.

\section{Ethical approval}

Ethics Committee approval was obtained from the UCT Faculty of Health Sciences Human Research Ethic Committee (HREC No. 065/2018).

\section{ORCID}

KJ Gqada (i) https://orcid.org/0000-0002-0684-7064

U Kotze (D) https://orcid.org/0000-0003-1405-474X

V Soldati (iD https://orcid.org/0000-0001-8577-6200

C Kloppers (iD https://orcid.org/0000-0003-2438-6879

J Krige (iD https://orcid.org/0000-0002-7057-9156

E Jonas (iD https://orcid.org/0000-0003-0123-256X

\section{REFERENCES}

1. Bray F, Ferlay J, Soerjomataram I, et al. Global cancer statistics 2018 - GLOBOCAN estimates of incidence and mortality worldwide for 36 cancers in 185 countries. CA Cancer J Clin. 2018;68(6):394-424. https://doi.org/10.3322/ caac. 21492.

2. Rawla P, Sunkara T, Gaduputi V. Epidemiology of pancreatic cancer: global trends, etiology and risk factors. World J Oncol. 2019;10(1):10-27. https://doi.org/10.14740/wjon1166.

3. McGuigan A, Kelly P, Turkington RC, et al. Pancreatic cancer - a review of clinical diagnosis, epidemiology, treatment and outcomes. World J Gastroenterol. 2018;24(43):4846-61. https://doi.org/10.3748/wjg.v24.i43.4846.

4. Fitzsimmons D, George S, Payne S, Johnson CD. Differences in perception of quality of life issues between health professionals and patients with pancreatic cancer. Psycho-oncology. 1999;8(2):135-43. https://doi.org/10.1002/(SICI)1099-1611(1 99903/04)8:2<135::AID-PON348>3.0.CO;2-Q.

5. Langenhoff BS, Krabbe PFM, Wobbes T, Ruers TJM. Quality of life as an outcome measure in surgical oncology. Br J Surg. 2001;88(5):643-52. https://doi.org/10.1046/j.1365-21 68.2001.01755.x.

6. Aaronson NK, Ahmedzai S, Bergman B, et al. The European Organization for Research and Treatment of Cancer QLQ-C30 - a quality-of-life instrument for use in international clinical trials in oncology. J Natl Cancer Inst. 1993;85(5):365-76. https://doi.org/10.1093/jnci/85.5.365.

7. Fitzsimmons D, Johnson CD, George S, et al. Development of a disease specific quality of life (QoL) questionnaire module to supplement the EORTC core cancer QoL questionnaire, the QLQ-C30 in patients with pancreatic cancer. Eur J Cancer. 1999;35(6):939-41. https://doi.org/10.1016/S09598049(99)00047-7.

8. Fitzsimmons D, Kahl S, Butturini G, et al. Symptoms and quality of life in chronic pancreatitis assessed by structured interview and the EORTC QLQ-C30 and QLQ-PAN26. Am J Gastroenterol. 2005;100(4):918-26. https://doi.org/10.1111/ j.1572-0241.2005.40859.x.

9. Guillemin F, Bombardier C, Beaton D. Cross-cultural adaptation of health-related quality of life measures literature review and proposed guidelines. J Clin Epidemiol. 1993;46(12):1417-32. https://doi.org/10.1016/0895-4356(93) 90142-N.

10. Kuliś D, Bottomley A, Velikova G, Greimel E, Koller M. EORTC quality of life translation procedure. 4 th ed. Brussels; 2017.

11. Harris PA, Taylor R, Thielke R, et al. Research electronic data capture (REDCap) - a metadata-driven methodology and workflow process for providing translational research 
informatics support, J Biomed Inform. 2009;42(2):377-81. https://doi.org/10.1016/j.jbi.2008.08.010.

12. Harris PA, Taylor R, Minor BL, et al. The REDCap consortium - building an international community of software platform partners. J Biomed Inform. 2019;95:103208. https://doi. org/10.1016/j.jbi.2019.103208.

13. Van Heijl M, Sprangers MA, De Boer AG, et al. Preoperative and early postoperative quality of life predict survival in potentially curable patients with esophageal cancer. Ann Surg Oncol. 2010;17(1):23-30. https://doi.org/10.1245/s10434009-0731-y.

14. Vanagas T, Gulbinas A, Johnson C, Pundzius J, Barauskas G. Translation and validation of the EORTC QLQ-PAN26 quality of life questionnaire for patients with pancreatic cancer. Acta Medica Litu. 2006;13(4):272-5. 